\title{
THE RATAN GALACTIC PLANE SURVEY
}

A Radio Continuum survey of the Galactic Plane with $343^{\circ} \geq l^{I I} \leq 19^{\circ}$ and $\left|b^{I I}\right|<5.5^{\circ}$ at $0.96,3.9$ and 11.2 GHZ

\section{S.A. TRUSHKIN}

Special Astrophysical Observatory of the Russian AS, RUSSIA

\section{Survey Description}

Now in radio continuum surveys more than 10,000 radio sources have discovered in the Milky Way plane but the Galactic origin only of a small part of them has been determined. The problem comes from the absence of estimates of source distance and the optical identification even for bright radio sources, and the most of sources have not spectral data at 2-3 frequencies. As followed some hundreds of sources have not classified as thermal or non-thermal. Now we don't know the full number of supernova remnants (SNRs) in the Galaxy. The simple estimates show that a sample of Galactic SNRs is not full as for weak and extended $\left(>15^{\prime}\right)$ as for bright and compact $\left(<3^{\prime}\right)$ SNRs (Trushkin 1993).

The three-frequency radio survey of the Galactic plane has been made at $0.96,3.9$ and $11.2 \mathrm{GHz}$ with the RATAN-600 radio telescope in three sets in 1991-1995 (Trushkin 1996b). The resolution (RA $\times$ DEC) is $4^{\prime} \times 75^{\prime}$, $1^{\prime} \times 39^{\prime}, 21^{\prime \prime} \times 14^{\prime}$ respectively. The noise level is typically 60,10 and 100 $\mathrm{mJy} /$ beam at $0.96,3.9$ and $11 \mathrm{GHz}$ respectively.

The total area of survey is near 400 degrees in square $(0.12 \mathrm{sr})$. It is one third of the volume of the Galaxy in which the outbursts of supernova are possible. The coordinate accuracy estimated on the comparison with Bonn and Texas surveys is better than $15^{\prime \prime}$ in R.A. Flux densities and spectral indices of near 40 known supernova remnants were measured (Trushkin 1996a).

The preliminary list includes more than 1500 radio sources. The crossidentification, comparison, spectral index studies have been made with 1800 selected sources from Effelsberg, Nabeyama, VLA, PMN, Texas radio surveys and from the IRAS catalog and another radio catalogs, included in radio astronomical database CATS (Verkhodanov \& Trushkin 1994, 1996). 


\section{Science from the Survey}

The search of the supernova remnants and variable radio sources has been made. Using the different criteria from the IRAS sources we searched the thermal sources: planetary nebulae and H II regions. We used ISSA (IPAC) infrared maps for comparison with the survey data. Some supernova remnant candidates have been found in survey area when we used IR/Radio criterion. G11.2-1.1 seems one of good SNR candidates.

The superluminal variable source and X-nova GRO J1655-40 has been detected (in 1991.2) in the survey at flux level $100 \pm 10$ at $3.9 \mathrm{GHz}$ far before the identification at radio wavelengths. The extended radio envelope has been detected around this source. Its spectrum is non-thermal $(\alpha=-0.6)$ and size is near $6^{\prime}$. Its origin could be connected either with associated supernova remnant or with blow-up envelope from central active source (Trushkin 1995).

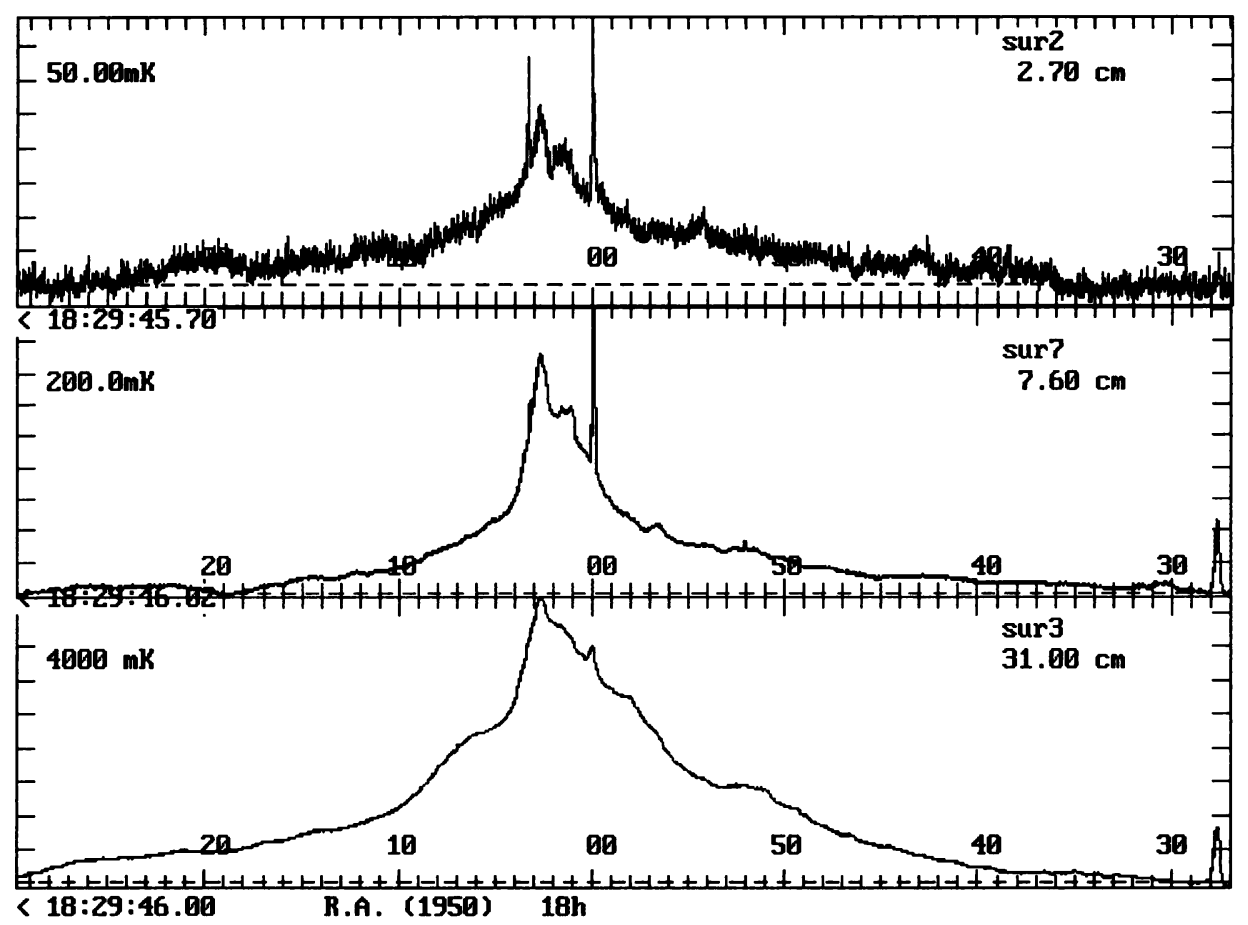

Figure 1. The survey cross-section of the Galaxy (Dec. $\left.=-21^{\circ} 40^{\prime},|b|<5.5\right)$ at three wavelengths. The extended galactic background is well seen on these scans.

The interesting point source $1820-239$ was identified in the confirmed shell SNR G8.7-5.0. It has a non-thermal spectrum, $\alpha=-0.4$ and size $<1^{\prime}$. The sequential sets of the Galactic survey in other epochs allowed to 
detect the variability of flux from this source on time scale of 3-4 months. Thus 1820-239 seems to be analogous to the Galactic binary systems such as SS433 and Cir X-1.

Then we have made the cross-identification of two catalogs: the Texas and IRAS-point source in the database CATS (Trushkin \& Verkhodanov 1995). 97 sources of the total 1208 pairs fall in the survey area. All 63 such sources with $\mathrm{S}_{365 M H z}>0.4 \mathrm{Jy}$ were detected at $3.9 \mathrm{GHz}$.

In Figure 1 the same cross-cuts of survey on DEC50: $-21^{\circ} 40^{\prime}$ at three wavelengths. The compact HII region 1800-217 and remnant of SN1604 (Kepler's SN) 1727-215, the latest from optically visible for last 400 years.

The work has been supported by the grant of the Russian Foundation of the Basic Researches N93-02-17086 and the travel RFBR grant N96-0227170. This report became also possible due to the hospitality of STScI and the SOC for IAU symposium 179.

\section{References}

Trushkin, S. A. 1993, in "XXV radio astronomical conference," Pushchino, FIAN, 76

Trushkin, S.A. 1994, Preprint of SAO, 107, 1

Trushkin, S. A. 1995, in "XXVI radio astronomical conference," St-Petersburg, IPA, 114 Trushkin, S.A. 1996a, Bulletin of SAO RAS, 41, 64

Trushkin, S.A. 1996b, Astron. Astrophys. Trans., 11, in press

Trushkin, S.A., \& Verkhodanov, O.V. 1995, Bulletin of SAO RAS, 39, 150

Verkhodanov, O. V., \& Trushkin, S. A. 1994, Preprint SAO RAS, N106, 1

Verkhodanov, O.V., \& Trushkin, S.A. 1996, Baltic Astronomy, in press 